\title{
Formalization of UML Composite Structure using Colored Petri Nets
}

\author{
Rao Sohail Iqbal ${ }^{1}$, Ramzan \\ Talib $^{2}$, Haseeb Ur Rehman ${ }^{4}$ \\ Department of Computer Science \\ Government College University \\ Faisalabad, Pakistan
}

\author{
Muhammad Awais ${ }^{3}$ \\ Department of Software Engineering \\ Government College University \\ Faisalabad, Pakistan
}

\author{
Wajid Raza ${ }^{5}$ \\ Department of Computer Science \\ NCBA\&E Lahore, Multan Campus \\ Multan, Pakistan
}

\begin{abstract}
Design specification and requirement analysis, during development process involved in transformation of real world problems to software system are subjected to severe issues owing to involvement of semantics. Though, for design and specification of object-oriented systems, Unified Modeling Language (UML) is now recognized as standard language however, its structures have numerous drawbacks which include lack of semantics definition and unidentified deadlocks. The research work proposes a model to avoid deadlocks, specifically in composite structure of UML. Verification of system models by formal methods holds significance, particularly, at requirement specification and design level, to ensure the accuracy of models and high light the design problems before implementation. The paper proposes the rules that allow software engineers to formalize the behavior of UML 2.0 composite structure using Colored petri nets. Using these rules, the research shall analyze the correspondent Colored petri nets and conclude the properties of the original work flow, using theoretical outcomes in the Colored petri nets domain.
\end{abstract}

Keywords-Design specification; UML (Unified Modeling Language); semantic; transformation; deadlocks

\section{INTRODUCTION}

Software engineering is facing multifarious challenges in the fields of design specification and requirement analysis owing to complications involved in system models' verification of real world thus rendering it a difficult task [1]. UML, governed by Open Group of Companies, is comparatively open standard, organized by the Open Management Group (OMG). It helps to make all important decisions of specification related to analysis, design and implementation. Though it is not visual programing, but its models are connected to a huge variety of programming languages [2]. It plays a vital role in design and implementation segment for building a software system. However, combination of a variety of object-oriented analysis and design methods into a single modeling language has inherited complicated issues [3]. Flaws in UML notation have invited the researchers to discover alternative methods for addressing the integration and compatibility issues with in order to allow an accurate and reliable design, modeling and development. UML has a set of standard symbols required for building objects and visualization of a software system, nonsoftware systems and business modeling [4]. It has become a standard for designing and implementation of object-oriented system [5], even though its semantics are semi-formal and liable to chances of ambiguities in the system design. Issues related to Modeling a system by UML are as under:

- Due to the graphical notation of UML structure it has certain chances of errors.

- At the design level of software system, hidden semantics of UML diagram can cause ambiguities.

- Model described by UML diagram can lead to multiple understandings, thereby disabling receivers of the model to take the precise decision with respect to the diagram.

The capability of UML can be enhanced by connecting it with formal methods and design the system by describing semantic rules in a formal way [6] owing to following reasons: $-$

a) Formal methods provide high level guarantee and reliability for analyzing of models.

b) It helps in detection of errors and defects at an early stage to reduce the cost.

c) Formal methods allow the developer to check model mathematically and prove its validity by the integration of UML diagrams with formal notations resulting in reliable, complete and accurate modeling of the system [7] [8].

UML 2.0, comprising integrated models and diagrams was introduced after success of UML 1.x. It allows us to model the structure of a system before its implementation, however significant problems related to identification of "errors" and "deadlocks" in designing phase persist [9] thereby leaving chances of system failure after execution. This research aims at resolution of this problem by transforming composite structure, using formal methods. Composite structure diagram enables us a deep insight of the class so as to what is actually happening as well as relationship of nested classes [10]. Using Colored petri nets model for transformation process which is a mathematical modeling (formal verification) language used for systems which have complex behavior. Since Colored petri nets are a formal model so they do not carry any ambiguity and can be validated [11]. The prominent benefit of this language which compelled us to deviate from traditional UML to Colored petri nets model is that it identifies the 'Errors' and 'Deadlocks' in a system before execution [9]. This will help to evaluate the model at an early stage thereby reducing the cost, 
risk and time span [12]. In the past few years a lot of work gone into formalization of UML diagrams, however composite structure diagram has failed to attract any worthwhile strength of researchers in the field. By transforming composite structure using Colored petri nets model will try to attain much precise results, while proving the scenario using some rules and formulate a case study towards the end.

\section{RELATED WORK}

A combination of $\mathrm{Z}$ notation and petri nets is proposed for designing safety critical systems. The part of construction cell is modeled and check its validity by safety interrelated properties [13]. A transformation from OCL (Object Constraint Language) into B is projected in [14]. Given transformation scheme is automatically derive to B except invariants of class. A UMC framework is present by the gatherings of UML state machine for the formal verification of concurrent systems. The formal model which is given in this system have transition having labels dually and uses logic to describe its properties comprising event-based and statebased logic. It allows user to see insights of a UML model to investigate behavioral aspects by visualization and model checking [15]. A framework of fuzzy logic is suggested to evaluate the compatibility of formalism on specific electronic commerce system. The evaluation of UML, Z notation, state charts, finite state machine (FSM) and Colored petri nets is carried out in combination with some domain by using the fuzzy logic structure and evaluation standards [16].

Raymond [17] shows the growing diversity of formal methods and mathematical models which plays a bridge role between "continuous" and "discrete" systems. A method is proposed by Than et al. [18] to formalize the syntax and semantics of UML state chart into $\mathrm{Z}$ notation, given semantics helps to elaborate the model consistency and assure its unambiguity and completeness. A formalization of higher order logic is presented based on arithmetic by using tool SPW (Signal Processing Work System) [19]. Akbarpour et al. uses the different rounding modes in fix point arithmetic like directed and even rounding modes. An analysis is performed to check the accuracy and basic arithmetic operations, multiplication, subtraction, addition and division.

Liu and Dong [20] give an algorithm to schedule the tasks executed in the finite set of memory, Input streams and code generated will be smaller in size as compared to previously used. Petri nets is used to do formal analysis and verification of the given algorithm. A simulation is projected by Hasan and Tahar [21] a higher order logic theorem for the analysis of critical parts and can give accurate results. A pair of probabilistic properties is also verified which was not evaluated by existing techniques. A methodology is offered to formalize some common diagrams of UML which are used in several phases of $\mathrm{Z}$ notation software development [22], a visual representation has been taken with this tool based approach by the formalization of UML diagrams. Derakhshandeh et al. [23] proposed a formal model for Access Control Policies (ACP) specification which have ability to express many ACPs, joining them in a unified model and also verifying conflicts among them.
Ma [24] presents the fuzzy extensions of ER/EER and UML models to manage a system at conceptual level. Some main notations have been prolonged and conforming the graphical notations, a formalization is performed by aiming on the basic concepts of class structure using simple case studies. A formal method is proposed based on mathematical mechanism to prove program correctness [25]. Algebra approach is used to improve the verification capability of the model which give the guarantee to provide error free model. Cunha et al. offered a method for transformation of UML sequence diagram to Petri nets to find out dead locks, errors and verifying liveness, reachability and safety of the model. This method is performed in an embedded control application, a sensory device which detect the object in the environment. A mathematical extension of OCL language is projected by cumulate its class library to deploy some mathematical notions including relations and functions [26]. Zhang and Liu [27] give a web-based service CCML (Cooperative Composition Modeling Language), is formalized by the formal verification method and also check its validity by proving a case study. A target fusion recognition method is proposed to check its reliability and validity based on the fuzzy sets and Petri nets under a complex environment which is also demonstrated by a simulation example [28]. UML activity model does not have firmly formal semantics, so it is hard to analyze them and activity model checking. An ontology-based method is presented for semantic checking of activity model which are divided into two parts dynamic semantics and static semantics [29].

\section{BACKGROUND}

In this section a brief discussion has been presented about the composite structure diagram and Colored petri nets.

\section{A. Composite Structure}

In UML 2.0 many diagrams were introduced which comprises Activity diagram, Class diagram, Sequence Diagram, Communication diagram, Component diagram, Composite Structure diagram, State diagram, Agent based UML and so on so forth. Composite structure diagram shows the internal structure of a specific classifier just like component diagram. A huge range of the notation of component diagram can be supported by composite structure diagram as well. Where the component diagram shows the internal structure, on the other hand composite structure shows the specific functionality of the classifier to be executed. It has four main parts which includes class, connectors, ports and interfaces. Class shows all the information, and composite structure diagram assumes to be complete when class diagram is considered as the smallest class structure which declares the given composite structure [30].

A connector is providing the interface between two objects. It might be an object which represent the relationship or a value hold in the variable. The value which is hold by the variable is the other way to represent the association between two objects. A port is a source which suggest the functionality of composite structure without exposing the internal operations. The port is a small square notation which is drawn above the edge line of the boarder. The multiplicity of port and its parent name are written near to it. The ports are 
connected with required/provided interfaces like from/to the situation. The required/ provided interfaces are commonly show with the socket/ball notation. Each port is connected to its internal operation using connectors and these internal operations can be some code kept by the classifier. The composite structure diagram and associated structures uses and semantics are well defined in [31][32][33] while the concepts of composition are effectively defined in [34][35].

\section{B. Petri Nets}

Petri Nets is a formal mathematical modeling language which is introduced in 1962 by Carl Adam Petri. It is used to formalize the models before implementation to identify the errors and deadlocks hence it can be rectified. It has strong formalized modeling capability in various fields like computer science and system engineering. It gives us facility to provide the mathematical theory with pictorial representation for a dynamic system. Mathematical theory will draw a precise model and let us know the behavior of system. And the graphical system shows the model visuals and state changes of the system. The combination of these two aspects are the key reasons of petri nets success. It has been used in modeling of many event driven systems such as computer networks, work flows, communication systems, real time computing systems, manufacturing plants, logistic networks and command and control systems [36].

Petri nets are the directed split graph having transitions and places which are represented by the nodes. Transitions are the events which may be occur and it is denoted by the bars and places are the conditions denoted by circles. Petri nets are consisting of three main parts which include places, transitions and arcs. Arcs are directed from places to transition or transition to places. They will never connect from place to place or transition to transition. The arc which comes out from place to transition that place will called transition input place, and arc which connects from transition to place is called transition output place. In the diagram of a petri nets, places are denoted by circles, transition are by long narrow rectangle and arcs are symbolized by one-way arrow which represent the relation from place to transition and transition to place [37].

\section{Composite Structure to Colored Petri Nets MODEL FORMALIZATION RULES}

In this segment, shown a briefly description about the transformation and formalization of some upper level operators which are offered in the UML2.0 composite structure into interactively equal Colored petri nets. For this purpose, here describe the semantics of operators, and explain how formalization will be accomplished. Furthermore, presented some outcomes by applying these concepts on a few demonstrative samples and also explain by a case study.

\section{A. Component}

In rule 1 , shown the trivial case where component $\mathrm{A}$ is implies the existence of class A. Now show its obtained colored petri nets fig. 1 .

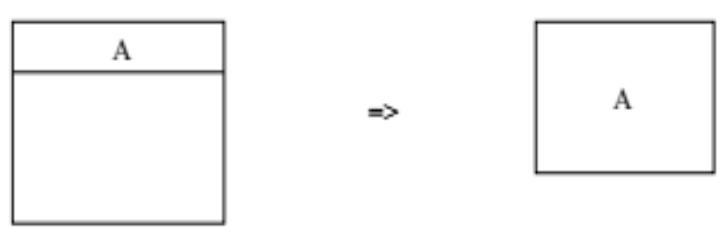

Rule 1 UML Composite Structure.

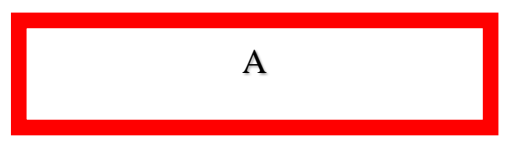

Fig. 1. Obtained CPN.

\section{B. Simple Composite Structure with Class into Component}

The case in rule 2 is denoted the situation of a simple composite structure A contains the B parts. This implies that class A having a sort of relationship with class B. When the relationship is executed, objects may communicate with each other by calling their operations. The part B is shown by solid rectangle with the composition of part $A$, though the relationship of $\mathrm{A}$ and $\mathrm{B}$ must be composite. A strong relationship shown by black diamond, which means that part $\mathrm{B}$ is life time owned by the parent object part A. Obtained CPN are shown in fig. 2 .

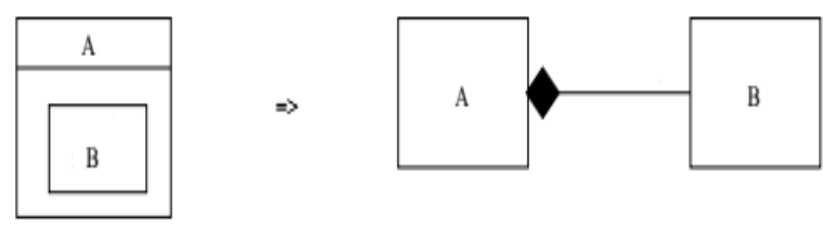

Rule 2. UML Composite Structure.

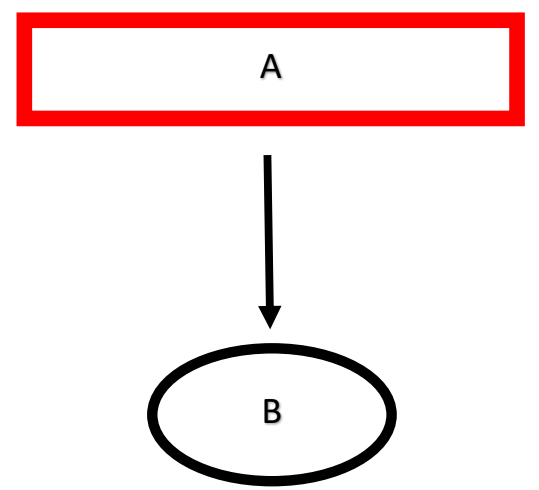

Fig. 2. Obtained CPN.

\section{Component with Port}

In composite structure a connection is made by port artifacts and signals send and receive via these ports. A trivial case of composite structure with port is shown in rule 3, which is also implied in class diagram. Connection between ports is termed as "wire", elaborate that a communication is occur by a signal sent from "wire" to receive port. Obtained Colored petri nets are given in fig. 3 . 


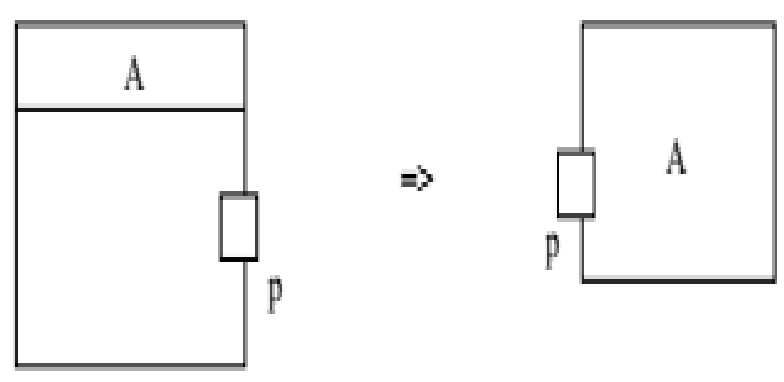

Rule 3. UML Composite Structure.
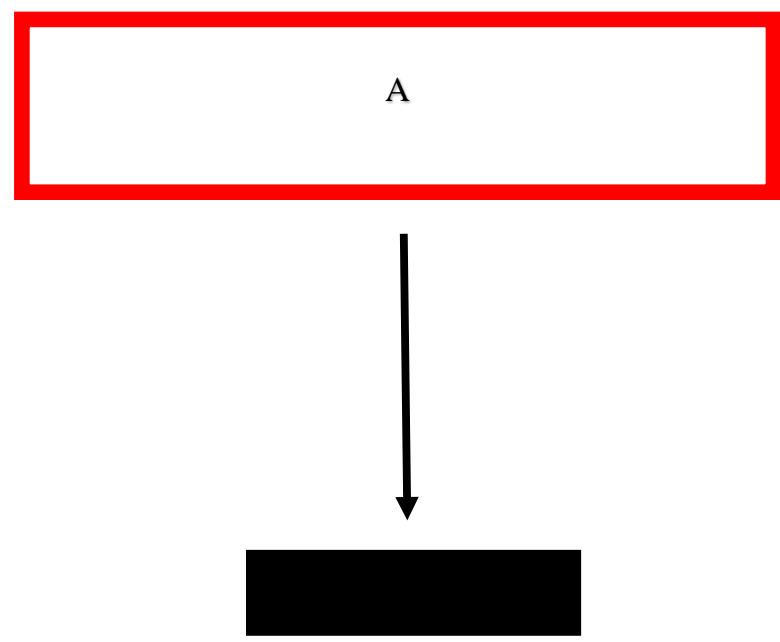

Fig. 3. Obtained CPN.

D. Two wired Ports

In this rule port $\mathrm{p}$ of $\mathrm{A}$ is by default visible and public to internal and external parts, although it is the parent class of $\mathrm{B}$ and $\mathrm{C}$ and port $\mathrm{q}$ and $\mathrm{r}$ visibility is internal and private because they are the child classes of A. Let us check its obtained CPN in fig. 4.
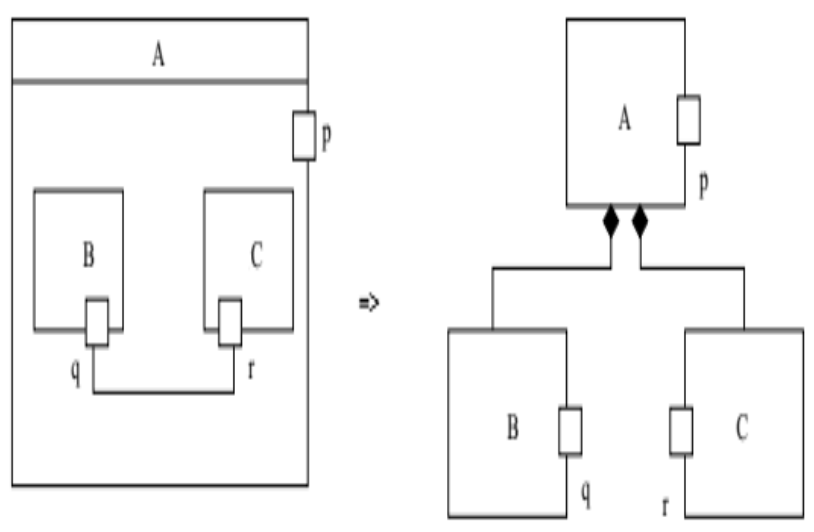

Rule 4. UML Composite Structure.

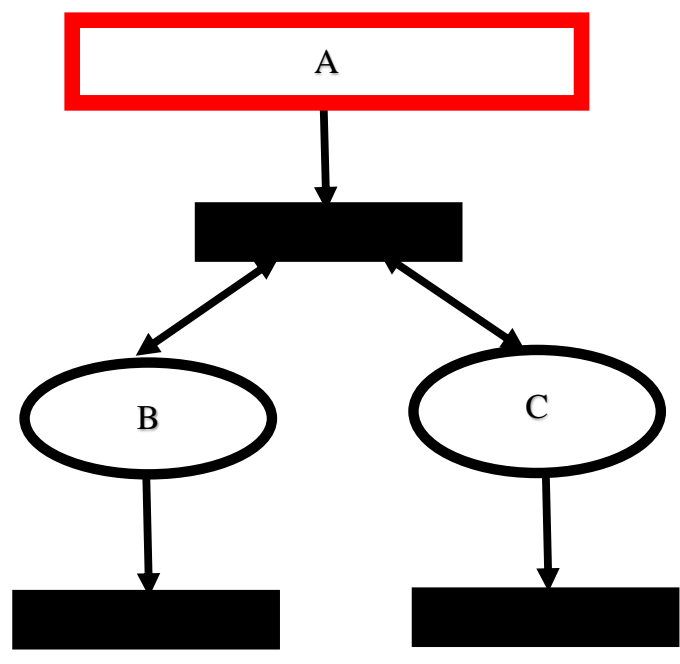

Fig. 4. Obtained CPN.

\section{E. Various Interface Combination}

If the interfaces are compatible, then two ports can communicate sensibly between required interface and provided interface. In rule 5 it is possible to establish connection in both directions for ports $\mathrm{p}$ and $\mathrm{q}$ as each either provided interface or required interface I and J. If connection for instance, between port $\mathrm{p}$ and port $\mathrm{r}$ are not sensibly connected to each other, no message will be understood and propagate with each other. And a meaningful communication will not take place as neither the required nor provided interface match. Obtained CPN are shown in fig. 5.
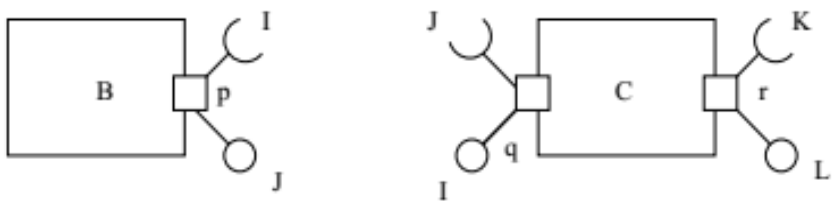

Rule 5. UML Composite Structure.

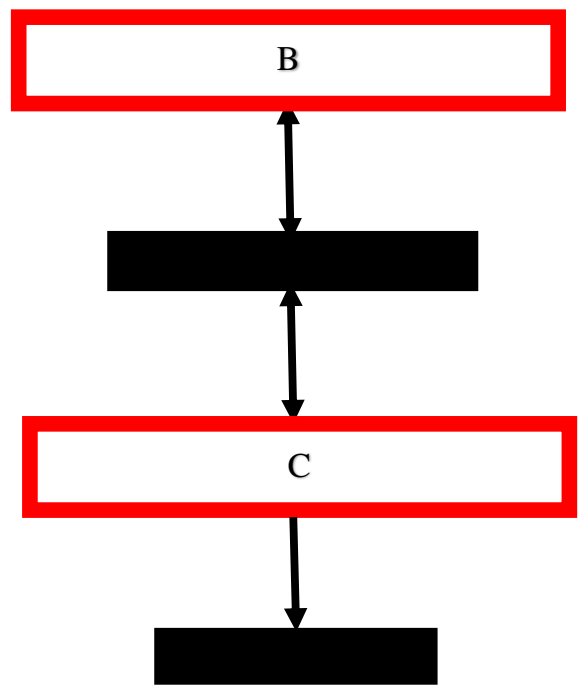

Fig. 5. Obtained CPN. 


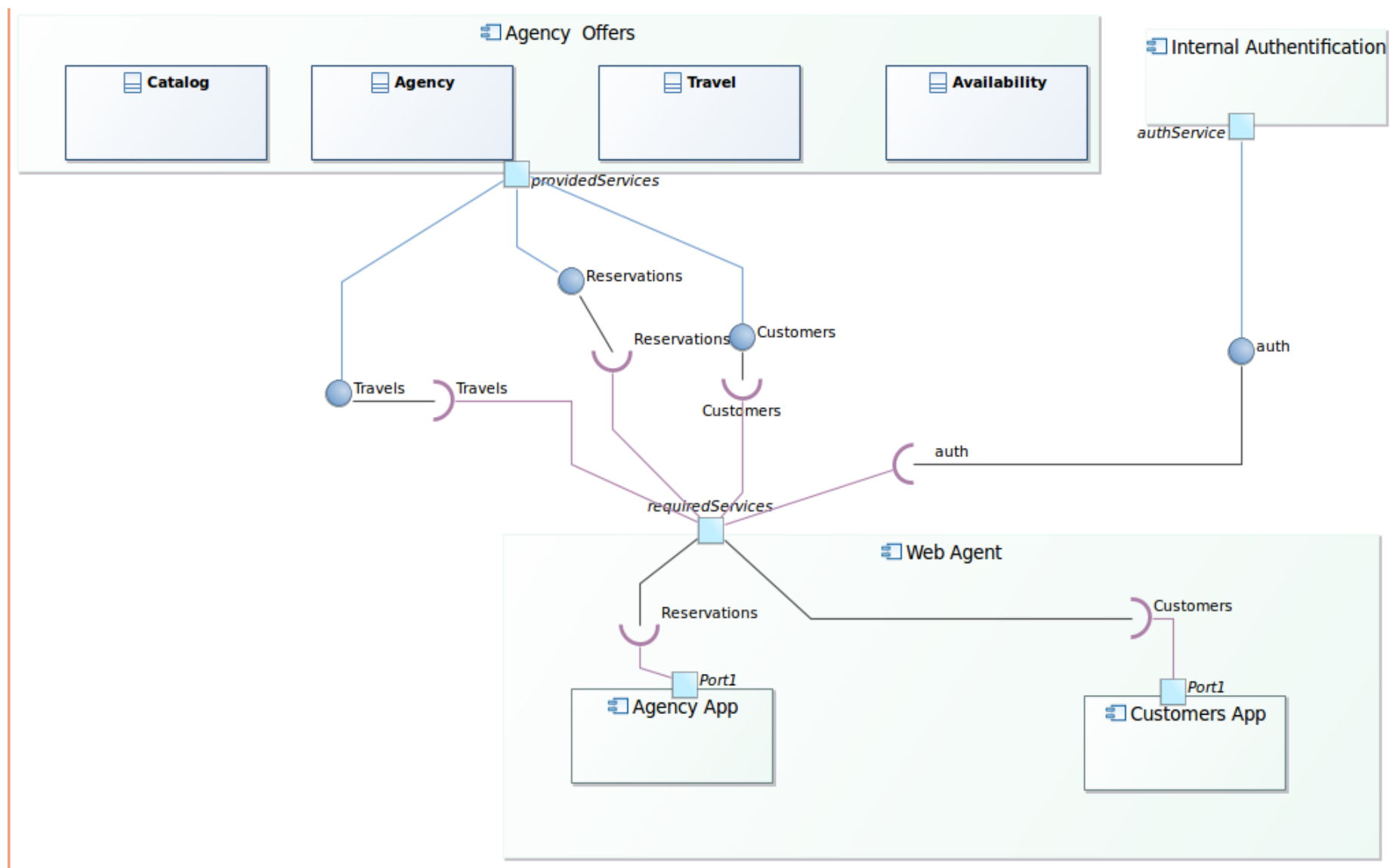

Fig. 6. Composite Structure Model of a Travel Agency.

The obtained petri nets elaborate the various connecting interfaces of a composite structure, where B is a class having both provided and required interface. After transition of class $\mathrm{B}$, another interface connecting the class $\mathrm{C}$ with provided interface.

\section{CASE STUdy}

By taking a real-world case study of a travel agency shown in fig. 6. We will prove the composite structure diagram by explaining each of its component. For this purpose, formalize a composite structure diagram into Colored petri nets model.

By taking a real-world case study of a travel agency formalization of composite structure will explain more clearly. In this case study proposed a structure of a travel agency that how it works with Web Agent, Agency Offers module and Internal Authentification. Furthermore, show the interactions between customer and agency also their relationship.

\section{A. Web Agent Module}

- There are two components to choose in the 'Web agent'.

- One is 'Customer app' for customer only and second is 'Agency app' for agency only.

- Each component has its connecting port with 'Required interfaces'.

The formalized web agent module is shown in fig. 9 .

\section{B. Agency Offers Module}

- There are four classes to access in the 'Agency offers'.

- One is 'Catalog' for agency catalog information and second is 'Agency' for Agency detail.

- Third is 'Travel' for getting information about services of travelling and fourth is 'Availability' to check the availability of flights.

- Each class and component have its connecting port with 'Provided interfaces

The formalized agency offers module is shown in fig. 8 .

\section{Internal Authentification}

- Before confirmation of flight an Internal Authentication of passenger is required after completing all formalities.

\section{FORMALIZED COLORED PETRI NETS MODEL}

A formalized colored petri nets model has been given below which shows the different operations performing in the model. In this model presented a web agent module taking the queries of the customer and forward it to the agency offers module and after internal authentification of the customer the process will place towards the final procedings. Fomalized model is shown in fig. 7 . 


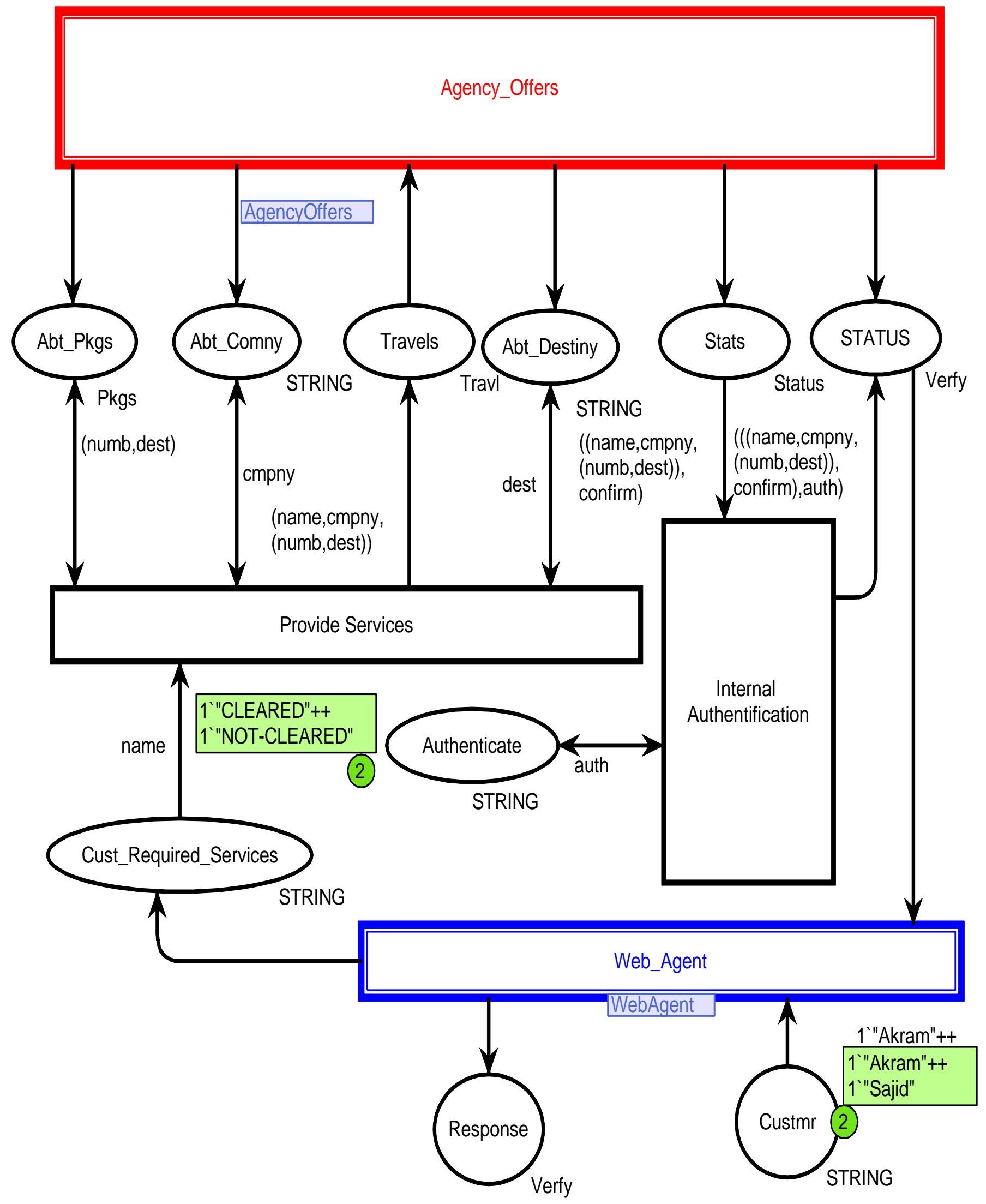

Fig. 7. The Agency Page - Architecture of CPN Model with Initial Marking $M_{0}$. 


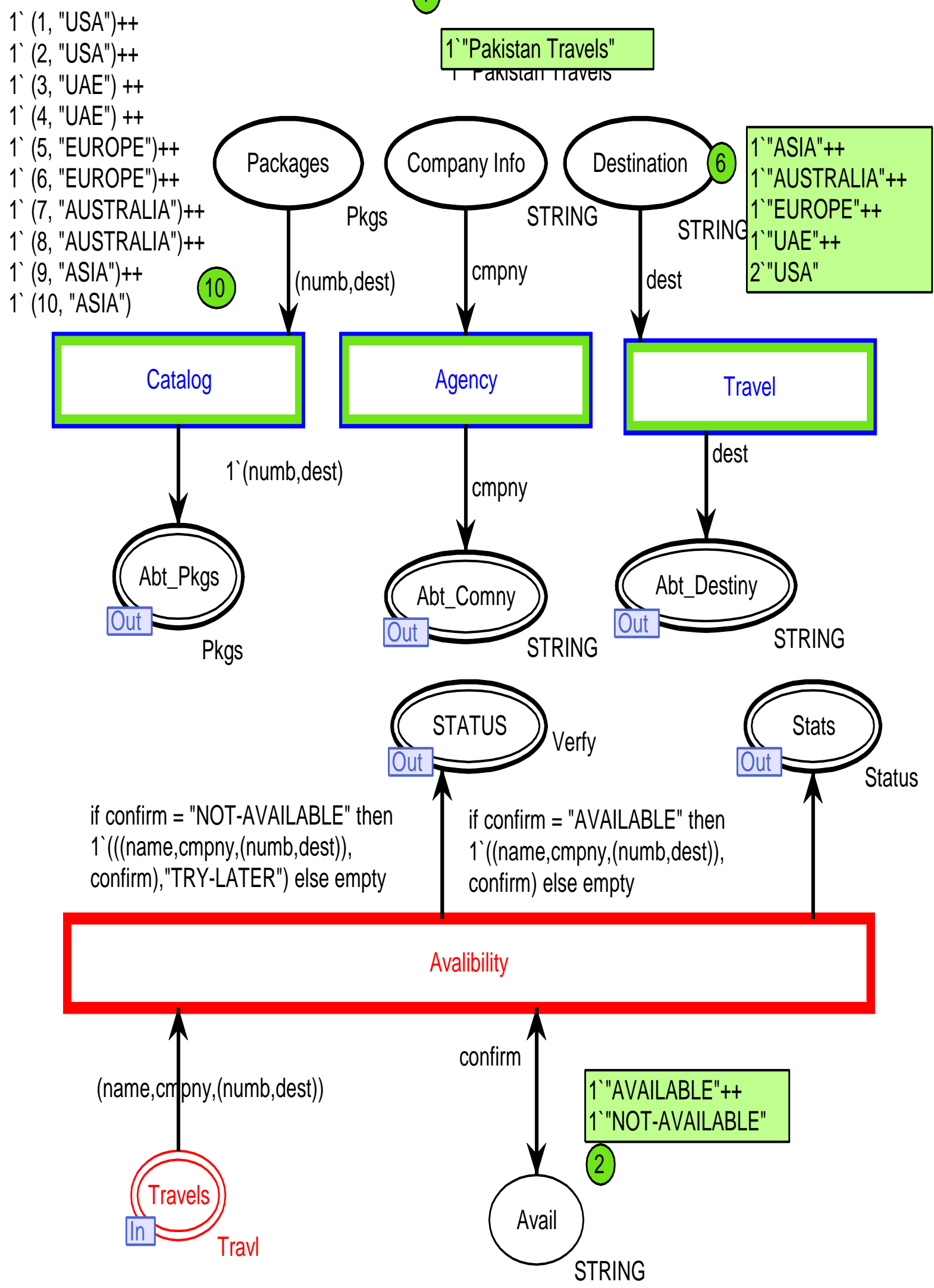

Fig. 8. The Agency Offers Module. 


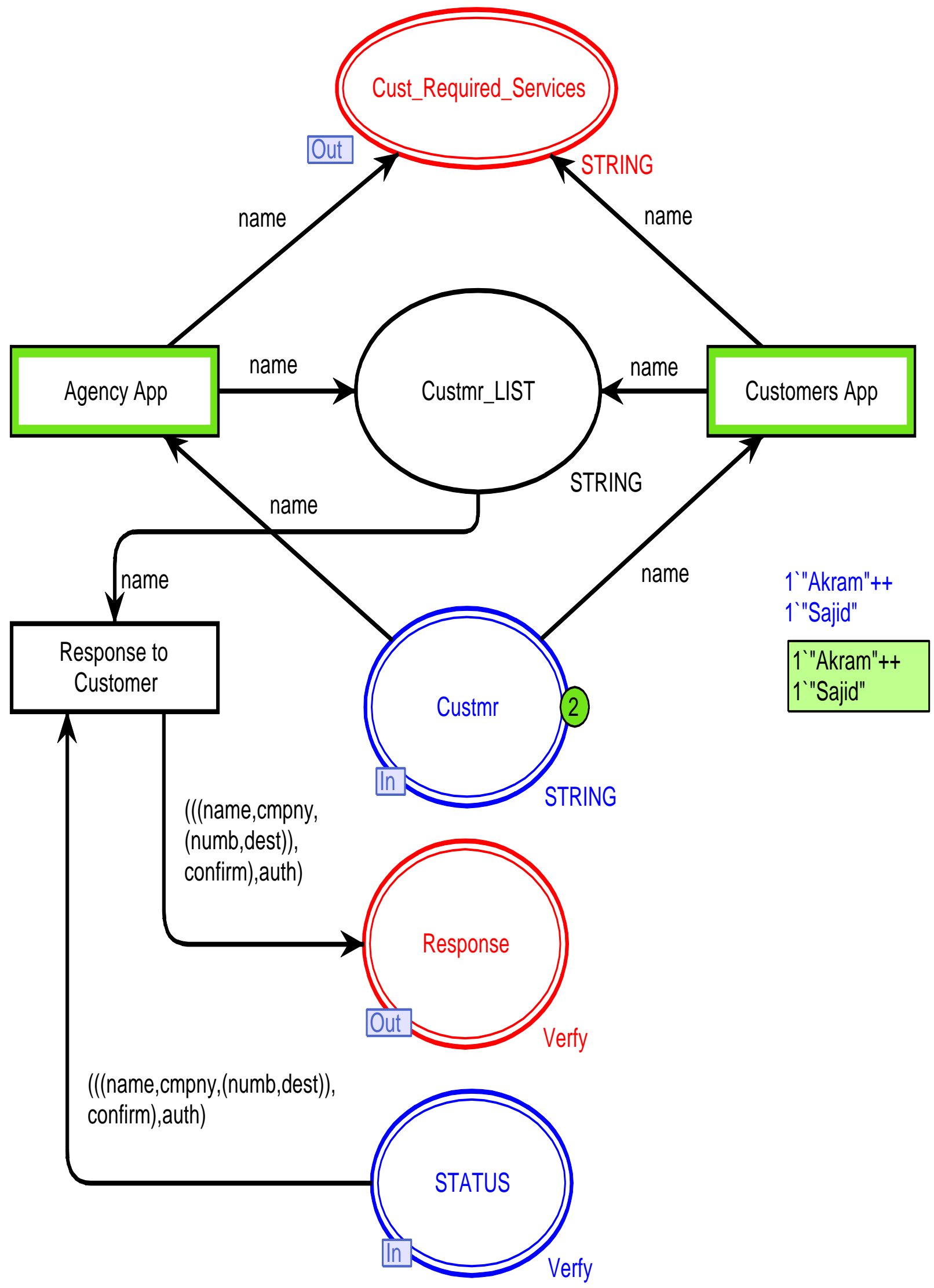

Fig. 9. The Web Agent Module. 


\section{VII.CPN TOOLs STATE SPACE REPORT}

CPN Tools state space report for:

/cygdrive/F/2 AFTER PhD 23.4.2018/RESEARCH PAPERS/GC FSD PAPER/TicktingHier.cpn Report generated: Sun May 13 13:56:09 2018

Statistics

State Space
Nodes: 20039
Arcs: $\quad 99589$
Secs: $\quad 300$
Status: Partial
Scc Graph
Nodes: 20039
Arcs: $\quad 99589$
Secs: $\quad 2$

Boundedness Properties

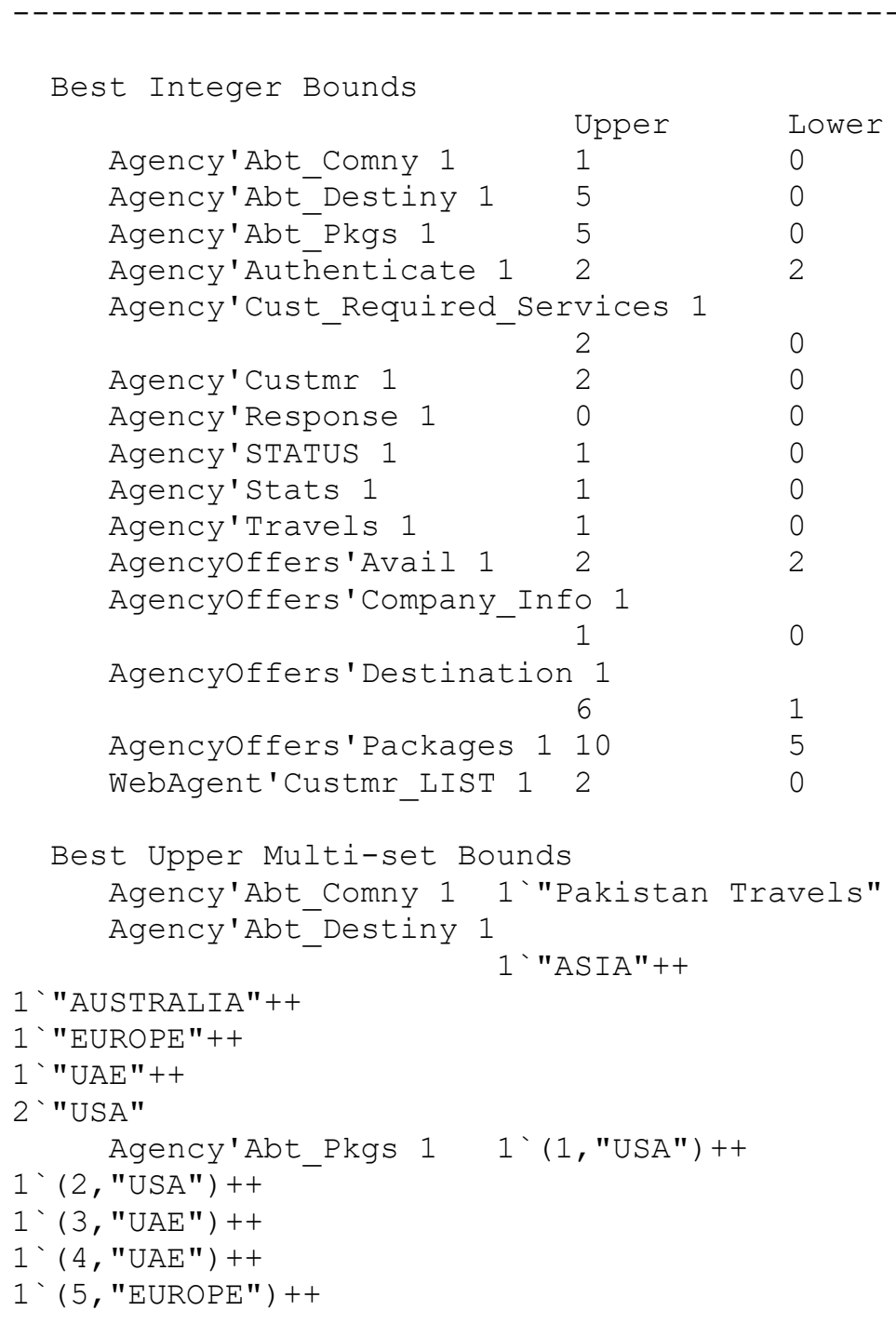


$1 `(6$, "EUROPE" $)++$

$1 \cdot(7$, "AUSTRALIA" $)++$

$1 \cdot(8, "$ AUSTRALIA" $)++$

$1 \cdot(9, " A S I A ")++$

1' $(10$, "ASIA")

Agency'Authenticate 1

1'"CLEARED"++

1 'NOT-CLEARED"

Agency'Cust_Required_Services 1

1' "Akram"++

1'"Sajid"

Agency'Custmr 1 1'"Akram"++

1 '"Sajid"

Agency'Response 1 empty

Agency'STATUS 1 1'(("Akram","Pakistan Travels", (2,"USA")), "NOT-

AVAILABLE"), "TRY-LATER") ++

1'( ( ("Akram", "Pakistan Travels", (4, "UAE")) , "NOT-AVAILABLE"), "TRY-LATER") ++

1`( ( ("Akram", "Pakistan Travels", (5, "EUROPE")) , "NOT-AVAILABLE") , "TRY-LATER" ) ++

1`( ("Akram", "Pakistan Travels", (6, "EUROPE")) , "NOT-AVAILABLE"), "TRY-LATER") ++

1'( ( "Akram", "Pakistan Travels", (7, "AUSTRALIA")), "NOT-AVAILABLE"), "TRY-LATER") ++

1'( ("Akram","Pakistan Travels", (10, "ASIA")) , "NOT-AVAILABLE"), "TRY-LATER")

Agency'Stats 1 1'( ("Akram", "Pakistan Travels", (2,"USA")), "AVAILABLE") ++

1`( ("Akram", "Pakistan Travels", (4,"UAE")), "AVAILABLE") ++

1`( ("Akram","Pakistan Travels", (5, "EUROPE")), "AVAILABLE") ++

1`( ("Akram", "Pakistan Travels", (6,"EUROPE")), "AVAILABLE") ++

1`( ("Akram","Pakistan Travels", (7, "AUSTRALIA")), "AVAILABLE") ++

1'(("Akram","Pakistan Travels", (10,"ASIA")), "AVAILABLE")

Agency'Travels 1 1'("Akram","Pakistan Travels", (1,"USA")) ++

1 ("Akram", "Pakistan Travels", (2, "USA")) ++

1`("Akram", "Pakistan Travels", (3,"UAE")) ++

1`("Akram","Pakistan Travels", (4, "UAE")) ++

1'("Akram","Pakistan Travels", (5, "EUROPE")) ++

1'("Akram","Pakistan Travels", (6, "EUROPE")) ++

1'("Akram","Pakistan Travels", (7, "AUSTRALIA")) ++

1' ("Akram","Pakistan Travels", (8, "AUSTRALIA")) ++

1`("Akram","Pakistan Travels", (9,"ASIA")) ++

1'("Akram","Pakistan Travels", (10,"ASIA")) ++

1'("Sajid","Pakistan Travels", (1,"USA")) ++

1'("Sajid","Pakistan Travels", (2,"USA")) ++

1`("Sajid","Pakistan Travels", (3, "UAE")) ++

1`("Sajid","Pakistan Travels", (4,"UAE")) ++

1'("Sajid", "Pakistan Travels", (5, "EUROPE")) ++

1'("Sajid","Pakistan Travels", (6, "EUROPE")) ++

1'("Sajid","Pakistan Travels", (7, "AUSTRALIA") ) ++

1'("Sajid","Pakistan Travels", (8,"AUSTRALIA")) ++

1'("Sajid","Pakistan Travels", (9,"ASIA")) ++

1'("Sajid","Pakistan Travels", (10,"ASIA"))

AgencyOffers'Avail 1

\section{1’"AVAILABLE"++}

1 'NOT-AVAILABLE"

AgencyOffers'Company Info 1

1'"Pakistan Travels"

AgencyOffers'Destination 1

1 ' $\mathrm{ASIA} "++$

1 ' "AUSTRALIA"++

1'"EUROPE"++

1 ' $\mathrm{UAE} "++$

2 ' "USA" 
AgencyOffers'Packages 1

$1 \cdot(2, " U S A ")++$

$1 \cdot(1$, "USA" ) ++

1 (3, "UAE") ++

1 ( 4, , $U A E ")++$

$1 \cdot(5$, "EUROPE" $)++$

1 (6, "EUROPE" ) ++

1 ( 7 , "AUSTRALIA") ++

1 ( 8, "AUSTRALIA" ) ++

$1 `(9$, "ASIA" ) ++

1'(10,"ASIA")

WebAgent'Custmr_LIST 1

1 'Sajid"

1'"Akram"++

Best Lower Multi-set Bounds

Agency'Abt_Comny 1 empty

Agency'Abt_Destiny 1

Agency'Authenticate 1

1 'CLEARED"++

1 'NOT-CLEARED"

Agency'Cust Required Services 1

empty

Agency'Custmr 1 empty

Agency'Response 1 empty

Agency'stATUS 1 empty

Agency'stats 1 empty

Agency'Travels 1 empty

AgencyOffers'Avail 1

1' "AVAILABLE"++

1 'NOT-AVAILABLE"

Agencyoffers'Company_Info 1

empty

AgencyOffers'Destination 1

empty

AgencyOffers'Packages 1

WebAgent'Custmr_LIST 1

empty

empty

Home Properties

Home Markings

None

Liveness Properties

Dead Markings

$13571[9999,9998,9997,9996,9995, \ldots]$

Dead Transition Instances

Agency'Internal_Authentification 1 


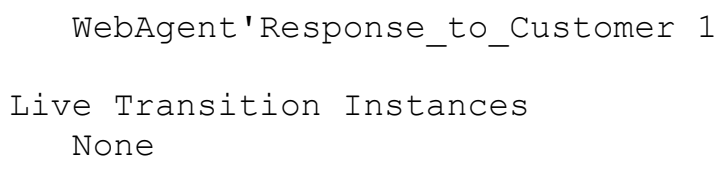

\section{CONCLUSION}

In this paper presented a bunch of rules and also perform a tool implementation to formalize the composite structure into equivalent colored petri nets. In UML 2.0, composite structure shows the internal structure of the classifier and give a deep insight view and cannot identify the errors and deadlocks with this. Proposed work explores the ways to identify the hidden errors and deadlocks at abstract level. This work is done with advance notation of petri nets, Colored petri nets gives more precise formalization. Hence the rules allow the formation of Colored Petri nets that shows various behavior of components in more accurate way.

\section{REFERENCES}

[1] N. H. Ali, Z. Shukur and S. Idris, "A Design of an Assessment System for UML Class Diagram," International Conference on Computational Science and Applications, Kuala Lampur, 26-29 August 2007, pp. 539546.doi:10.1109/ICCSA.2007. 31.

[2] UML Distilled Third Edition June 2003, pp. 118.

[3] Review and analysis of the issues of Unified Modeling Language for Visualizing, Specifying, Constructing and Documenting the Artifacts of a Software-Intensive System by Dr.S.S.Riaz Ahamed (2009).

[4] A Critical Analysis and Treatment of Important UML Diagrams Enhancing Modeling Power by Fahad Alhumaidan (2012).

[5] K. E. Hamdy, M. A. Elsoud and A. M. El-Halawany, "UML-Web Engineering Framework for Modeling Web Application," Journal of Software Engineering, Vol. 5, No. 2, 2011, pp. 49-63. doi:10.3923/jse.2011.49.63.

[6] X. He, "Formalizing UML Class Diagrams: A Hierarchical Predicate Transition Net Approach," Proceedings of Twenty-Fourth Annual International Computer Software and Applications Conference, Taipei, 25-27 October 2000, pp. 217-222. doi:10.1109/CMPSAC.2000. 884721.

[7] M. Shroff and R. B. France, "Towards Formalization of UML Class Structures in Z," 21st International Conference on Computer Software and Applications, Washington DC, 1-15 August 1997, pp. 646-651.

[8] Formal methods by the institution of Engineering and Technology (2011), pp. 4.

[9] A. M. Mostafa, A. I. Manal, E. B. Hatem and E. M. Saad, "Toward a Formalization of UML2.0 Meta-Model Using Z Specifications," Proceedings of 8th ACIS International Conference on Software Engineering, Artificial Intelligence, Networking and Parallel/Distributed Computing, Qingdao, 30 July-1 August 2007, pp. 694701 doi:10.1109/SNPD. 2007.508.

[10] E. Cunha, M. Custodio, H. Rocha and R. Barreto, "Formal Verification of UML Sequence Diagrams in the Embedded Systems Context," Brazilian Symposium on Computing System Engineering (SBESC), 2011, pp. 39-45.

[11] Composite structure \& Component diagrams by Greg Guyles csci5448 Prof. Anderson (2012).

[12] Transformation Of Uml Activity Diagrams Into Petri Nets For Verification Purposes by Bhawana Agarwal (2013).

[13] W. S. Changchien, J. J. Shen and T. Y. Lin, "A Preliminary Correctness Evaluation Model of Object-Oriented Software Based on UML," Journal of Applied Sciences, Vol. 2, No. 3, 2002, pp. 356-365. doi:10.3923/jas.2002.356.365.

[14] M. Heiner, and M. Heisel, "Modeling Safety Critical Systems with Z and Petri-Nets," Proceedings of International Conference on Computer Safety, Reliability and Security, Toulouse, 27-29 September 1999, pp. 361-374.

[15] Leading and J. Souquieres, "Integration of UML and B Specification Techniques: Systematic Transformation from OCL Expressions into B," Proceedings of 9th Asia-Pacific Software Engineering Conference, Gold Coast, 4-6 December 2002, p. 495.

[16] H. Beek, A. Fantechi, S. Gnesi and F. Mazzanti, "State/Event-Based Software Model Checking," Proceedings of 4th International Conference on Integrated Formal Methods, Canterbury, 4-7 April 2004, pp. 128-147.

[17] S. A. Ehikioya and B. Ola, "A Comparison of Formalisms for Electronic Commerce Systems," Proceedings of International Conference on Computational Cybernetics, Vienna, 30 August-1 September 2004, pp. 253-258. doi:10.1109/ICCCYB. 2004.1437721.

[18] T. B. Raymond, "Integrating Formal Methods by Unifying Abstractions," Springer, Berlin, 2004, pp. 441-460.

[19] X. Than, H. Miao and L. Liu, "Formalizing Semantics of UML Statecharts with Z," Proceedings of 4th International Conference on Computer \& Information Technology, Wuhan, 14-16 September 2004, pp. 1116-1121. doi:10.1109/ CIT.2004.1357344.

[20] B. Akbarpour, S. Tahar and A. Dekdouk, "Formalization of Cadence SPW Fixed-Point Arithmetic in HOL," Formal Methods in System Design, Vol. 27, 2005, pp. 173-200.

[21] C. Liu and X. M. Dong, "An Improved Quasi-Static Scheduling Algorithm for Mixed Data-Control Embedded Software," Journal of Applied Sciences, Vol. 6, No. 7, 2006, pp. 1571-1575.

[22] O. Hasan and S. Tahar, "Verification of Probabilistic Properties in the HOL Theorem Prover," Proceedings of the 6th International Conference of Integrated Formal Methods, Oxford, 2-5 July 2007, pp. 333-352.

[23] S. Sengupta and S. Bhattacharya, "Formalization of UML Diagrams and Consistency Verification: A Z Notation Based Approach," Proceedings of India Software Engineering Conference, Hyderabad, 19-22 February 2008, pp. 151-152. doi:10.1145/ 1342211.1342248.

[24] Z. Derakhshandeh, B. T. Ladani and N. Nematbakhsh, "Modeling and Combining Access Control Policies Using Constrained Policy Graph (CPG)," Journal of Applied Sciences, Vol. 8, No. 20, 2008, pp. 35613571. doi:10.3923/jas.2008.3561.3571.

[25] Z. M. Ma, "Fuzzy Conceptual Information Modeling in UML Data Model," International Symposium on Computer Science and Computational Technology, Shanghai, 20-22 December 2008, pp. 331334. doi:10.1109/ ISCSCT.2008.353.

[26] C. Yong, "Application of Wu's Method to Proving Total Correctness of Recursive Program," Information Technology Journal, Vol. 9, No. 7, 2010, pp. 1431-1439. doi:10.3923/ itj.2010.1431.1439.

[27] M. T. Bhiri, K. Mourad, M. Graiet and P. Aniorte, "UML/ OCL and Refinement," 18th IEEE International Conference and Workshops on Engineering of Computer-Based Systems, Las Vegas, 27-29 April 2011, pp. $14-158$.

[28] X. G. Zhang and H. Liu, "Formal Verification for CCML Based Web Service Composition," Information Technology Journal, Vol. 10, No. 9, 2011, pp. 1692-1700. doi :10.3923/ itj.2011.1692.1700. 
[29] Z. Shi, "Intelligent Target Fusion Recognition Based on Fuzzy Petri Nets," Information Technology Journal, Vol. 11, No. 4, 2012, pp. 500503. doi:10.3923/itj.2012.500.503.

[30] Z. X. Wang, H. He, L. Chen and Y. Zhang, "Ontology Based Semantics Checking for UML Activity Model," Information Technology Journal, Vol. 11, No. 3, 2012, pp. 301-306. doi:10.3923/itj. 2012.301.306.

[31] McCarthy, J.: Circumscription - a form of non-monotonic reasoning. Artificial Intelligence 13 (1980) 27-39.

[32] Haugen, Ø., Møller-Pedersen, B., Weigert, T.: Structural modeling with uml 2.0, classes, interactions and state machines. In Lavagno, L., Martin, G., Selic, B., eds.: UML for Real, Design of Embedded Real-Time Systems. Kluwer AcademicPublishers (2004) 53-76.
[33] Bock, C.: Uml 2 composition model. Journal of Object Technology 3(10) (2004) 47-74.

[34] Hofmeister, C., Nord, R., Soni, D.: Applied Software Architecture. Addison-Wesley (1999) 0201325713.

[35] Bock, C., Odell, J.: A foundation for composition. Journal of Object Oriented Technology 7(6) (1994) 10-14

[36] Odell, J.: Advanced Object-Oriented Analysis and Design Using UML. SIGS Reference Library. Cambridge (1998) 0-521-64819-X.

[37] Petri Nets for Dynamic Event-Driven System Modeling by Jiacun Wang, Department of Software Engineering, Monmouth University, West Long Branch, NJ 07764 (2012). 